\title{
Spreading of Lipid Monolayers on Hydrophilic Substrates at Increased Relative Humidities
}

\author{
Tobias Baumgart and Andreas Offenhäusser*
}

\author{
Max-Planck Institute for Polymer Research, Ackermannweg 10, D-55128 Mainz, Germany
}

Received J anuary 22, 2002. In Final Form: April 25, 2002

\begin{abstract}
A self-repairing ability of phospholipid monolayers deposited onto thin hydrogel films was observed by fluorescence mi croscopy, in terms of the expansion and lateral spreading of themonol ayer intofilm defects. The spreading was quantitatively analyzed by covering half of a substrate supporting a thin hydrogel film with a lipid monol ayer by Langmuir-B lodgett transfer and observing thespreading of theinitially confined monol ayer at increased relative humi dities due to the hydration of both lipid headgroups and the polymer support. During the observation time of a typical spreading experiment, a constant spreading vel ocity was observed. A strong influence of monolayer pressure on the spreading velocity was observed. A nonlinear relationship between spreading vel ocity and monol ayer pressure was found, which can possibly beexplained by a dependence of thedisjoining pressurein the monol ayer/substrateinterfaceon themonolayer pressure. The spreading velocity was additionally influenced by the kind of support and by the nature (e.g., phase state) of the deposited monolayer.
\end{abstract}

\section{Introduction}

The phospholipid monolayer represents half of the bilayer, which is regarded to constitute, among other components, the compartmentalizing part of biological membranes. $^{1}$

While the physicochemical parameters of phospholipid monolayers (more generally, Langmuir monolayers) at the air/water interface have been systematically and intensively studied for several decades ${ }^{2}$ and arecurrently relatively well understood, ${ }^{3}$ studies of substrate-supported phospholipid monolayers (more generally, LangmuirBlodgett (LB) films) are relatively scarce ${ }^{4}$ sofar. H owever, recently interest has particularly grown, partially due to upcoming technical applications ${ }^{5}$ and partially dueto the invention of new approaches, especially thescanning probe techniques ${ }^{6}$ such as STM (scanning tunneling microscopy), ${ }^{7}$ AFM (atomicforcemicroscopy), ${ }^{8} \mathrm{NSOM}$ (near-field scanning optical microscopy), ${ }^{9}$ and related methodol ogies. Interest has especially been paid to the microscopic structure of supported phospholipid films. Both the film morphology directly after LB transfer and lateral re organizations upon storage have been examined. $8,10 \mathrm{~F}$ or the latter to take place, it has been observed that an increased relative humidity $(\mathrm{RH})$ or the presence of other solvents in the gas phase is favorable. ${ }^{10-13}$

* To whom correspondence should be addressed at the Institute for Thin Films \& Interfaces (ISG2), Forschungszentrum J ülich, D-52425] ülich, Germany. Phone: +49-2461-2330. Fax: +49-246161-2333. E-mail: a.offenhaeusser@fz-juelich.de.

(1) Singer, S. J .; Nicholson, G. L. Science 1972, 175, 720-731.

(2) Möhwald, H. Annu. Rev. Phys. Chem. 1990, 41, 441-476.

(3) McConnell, H. Annu. Rev. Phys. Chem. 1991, 42, 171-195.

(4) Fischer, A.; Sackmann, E. J . Phys. (Paris) 1984, 45, 517-527.

(5) Swalen, J . D.; Allara, D. L.; Andrade, J . D.; Chandross, E. A.; Garoff, S.; I sraelachvili, J .; McCarthy, T. J .; Murray, R.; Pease, R. F.; Rabolt, J. F.; Wynne, K. J .; Yu, H. Langmuir 1987, 3, 932-950.

(6) I vanov, G. R.; Petkova, J . I.; Okabe, Y.; Aoki, D.; Takano, H.; Kawate, H.; Fujihira, M. Supramol. Sci. 1997, 4, 549-557.

(7) Smith, D. P. E.; Bryant, A.; Quate, C. F.; Rabe, J . P.; Gerber, Ch.; Swallen, J. D. Proc. Natl. Acad. Sci. U.S.A. 1987, 84, 969.

(8) Chi, L. F.; Anders, M.; Fuchs, H.; J ohnston, R. R.; Ringsdorf, H . Science 1993, 259, 213-216.

(9) Hwang, J.; Tamm, L. K.; Böhm, C.; Ramalingam, T. S.; Betzig, E.; Edidin, M. Science 1995, 270, 610-614.

(10) Shiku, H.; Dunn, R. C.J . Phys. Chem. B 1998, 102, 3791-3797.
However, "surprisingly few studies have been made on the effects of humi dity or other vapors on monolayer-coated surfaces exposed to ambient conditions". ${ }^{11}$ Moreover, even the few works available in most cases only qualitatively describe the effects of changing the ambient humidity; quantitative assessments of the effect of $\mathrm{RH}$ on dynamic parameters of supported phospholipid monolayers are rare. ${ }^{14}$

Generally, biosensor applications based on model membrane systems demand a highly insulating membrane. ${ }^{15}$ The substrate-supported lipid monolayer represents an intermediate stage in the fabrication of functionalized model membrane systems and therefore needs to be properly understood. Additionally, the monolayer stage all ows for physi cochemical studies, which would be more complicated, if not impossible, in the case of a lipid bilayer.

The structure of an initially deposited monolayer significantly influences the characteristics of the second monolayer deposited onto the first, ${ }^{16}$ and therefore determines the quality of the resulting bilayer. Several processes can lead tofilm defects. Monolayer qual ity might be reduced due to mechanical distortions during the transfer process. Furthermore, deposition by LangmuirBlodgett transfer inevitably comprises a drying step. If a polymer cushion is used as a membranesupport, ${ }^{17}$ during the drying step, thinning of the cushion might result in local, transient area changes, which could be inscribed into the lipid layer permanently dueto reduced dynamics of lipid molecules in the dry state.

Obviously, an increased humidity will lead to a reduced motional coupling of thelipid layer tothehydrogel support,

(11) Chen, Y. L. E.; Gee, M. L.; Holm, C. A.; Israelachvili, J . N.; McGuiggan, P. M. J . Phys. Chem. 1989, 93, 7057-7059.

(12) Chi, L. F.; J ohnston, R. R.; Ringsdorf, H. Langmuir 1992, 8, $1360-1365$.

(13) Berman, A. D.; Cameron, S. D.; I sraelachvili, J . N.J . Phys. Chem. B 1997, 101, 5692-5697.

(14) Baumgart, T.; Offenhäusser, A. Submitted for publication.

(15) Stelzle, M.; Weissmüller, G.; Sacmann, E.J . Phys. Chem. 1993, 97, 2974-2981.

(16) Merkel, R.; Sackmann, E.; Evans, E. J . Phys. (Paris) 1989, 50, 1535-1555.

(17) Sackmann, E. Science 1996, 271, 43-48. 
which leads toenhanced diffusion and increased spreading velocity. Positive influences of the exposure of the monolayer to increased humidities are possibly the leveling out of local density gradients and the healing of voids or cracks in themonolayer film. Thelatter twotypes of defects have been observed by el ectron microscopy ${ }^{4}$ and scanning probemicroscopy techniques; see, e.g., ref 18. The healing of monolayer defects after the monolayer mobility is increased can be assumed to be due to either surface diffusion or a collective transport by spreading, or a combination of both transport mechanisms. ${ }^{19}$

Thetopic of the present work wastostudy the possibility of phospholipid monolayer spreading and the spreading characteristics upon exposureto a high ambient humidity. Monolayers were deposited onto thin, water-swellable polysaccharide films to reduce the frictional coupling between the monolayer and the underlying substrate. For comparison, monolayers were also transferred to and analyzed on glass supports. Theinfluence of thesubstrate, the humidity, and the lateral pressure within the monolayer on the spreading behavior was analyzed.

The spreading of monomol ecular amphiphile films on solids was analyzed in refs 20-23. Generally, a square root behavior of precursor film radius growth with a very sharp spreading front and a dense monolayer was found. Studies on the spreading behavior of trisiloxane oligo(ethylene oxide) surfactants revealed that the ambient humidity influenced the spreading rates remarkably.

The spreading of phospholipid membranes on solid substrates thus far has been analyzed in terms of the spreading of bilayers deposited onto hydrophilic substrates. ${ }^{24,25}$ The spreading of a single bilayer has been observed, with a spreading front displacement following a square root behavior with respect to the observation time.

Thespreading of L angmuir-Blodgett monol ayers (and of phospholi pid monol ayers in particular) on sol id supports to the best of our knowledgeso far has not been examined. This might partially be due to the fact that spreading on typical supports such as silicon oxide, glass, or mica is too slow. To circumvent this problem, in the present study, a water-swellable hydrogel was used for supporting the monolayer.

\section{Methods and Materials}

Fluorescence Microscopy. Fluorescence microscopy was performed with an inverted microscope (IX 70, Olympus, Hamburg, Germany). A high-pressure mercury burner (HBO 100) served as light source. A fluorescence cube consisting of an excitation band-pass filter (BP 470-490, Olympus) and a barrier filter (BA 515, fluorescence cube U-MNIB, Olympus) was used. The lipid monolayer was observed through a transparent glass substrate (Mettler Glas, Rettberg, Göttingen, Germany; length $3.2 \mathrm{~cm}$, width $2.6 \mathrm{~cm}$, thickness $150-180 \mu \mathrm{m}$ ) by means of an oil immersion objective (100x, NA $=1.3$; Zeiss, Göttingen). The substrate was mounted into a copper chamber (Figure 1), which allowed for thecontrol of temperatureand $\mathrm{RH}$ as described below. All spreading experiments werecarried out at room temperature, i.e., $25^{\circ} \mathrm{C}$.

(18) Sikes, H. D.: Woodward, J. T.; Schartz, D. K. J . Phys. Chem. 1996, 100, 9093-9097.

(19) Gaver, D. P.; Grotberg, J . B. J . Fluid Mech. 1990, 213, 127-148.

(20) Tiberg, F.; Cazabat, A.-M. Langmuir 1994, 10, 2301.

(21) Tiberg, F.; Cazabat, A.-M. Europhys. Lett. 1994, 25, 205-210.

(22) Villette, S.; Valignat, M. P.; Cazabat, A. M.; J ullien, L.; Tiberg, F. Langmuir 1996, 12, 825-830.

(23) Lucht, R.; Bahr, C. Phys. Rev. Lett. 2000, 85, 4080-4083.

(24) Rädler, J .; Strey, H.; Sackmann, E. Langmuir 1995, 11, 45394548.

(25) Nissen, J .; Gritsch, S.; Wiegand, G.; Rädler, J . O. Eur. Biophys. J ., B 1999, 10, 335-344.

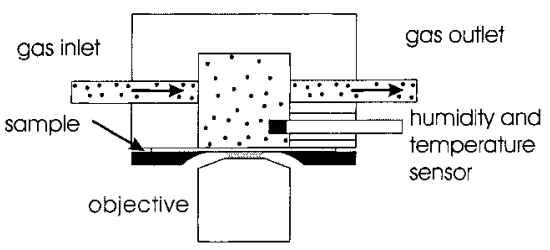

Figure 1. Humidity chamber for microscopic observation of supported lipid monolayers. The chamber walls were temperature-controlled by a water circle. The temperature and humidity sensor was thermally isol ated from thechamber walls by a Teflon capsule. Prior to the objective being glued to the sample backside (microscopic coverglass), lipid on thebackside of the coverglass was thoroughly wiped off with 2-propanol.

Humidity Adjustment. Spreading experiments were performed in a temperature and humidity-controlled atmosphere. Lipid monolayers were end osed in a copper chamber, which was sealed by the glass substrate (Figure 1 ). The $\mathrm{RH}$ inside the chamber was adjusted by the mixing of a dry and a watersaturated gas stream. ${ }^{26}$ The dry gas was obtained by passing a nitrogen gas stream through a series connection of two silicagel-filled bottles. The water-rich gas was produced by bubbling nitrogen through a series connection of two water-filled bottles. Computer-controlled valves (type 1259C-10000SV, MKS Instruments, Germany) allowed for changing the mixing ratio at a constant total flow. Carewas taken to avoid water droplets from creeping along the gas tubes and entering the measurement chamber. Therefore, a water trap was inserted into the wet gas tube and the final part of the mixed gas tube was filled with cotton wool. Therelativehumi dity was determined by measuring both thetemperatureand gas-phase water content in the chamber by a sensor, which was placed cl osetothesubstrate. Thehumidity and temperature sensor was a Hygroclip miniature air probe with an AlH interface, Rotronic, Germany. Thetransducer was equipped with an RS 232 interface, which enabled data readout by a PC. A feedback loop allowed for adjusting RH tothe desired value.

Langmuir-Blodgett Transfer. The Langmuir-Blodgett method was used for preparing supported lipid monolayers. A trough with a mechanical dipper and Wilhel my balancewas used (NI MA Technol ogy Ltd., Coventry, U.K.). Thesubphaseconsisted of ion-exchanged Millipore filtered water (Millipore Milli-Q system, $R=18.2 \mathrm{M} \Omega \mathrm{cm}$ ). To cover only half of the substrate area with a lipid monolayer, the substrates were immersed halfway into the subphase ${ }^{13}$ before transfer. Prior to spreading of the lipid solution, the polymer-covered substrates were equilibrated in the trough (for approximately $1 / 2 \mathrm{~h}$ ) to al low for a smooth three-phase line to be established. Omitting the latter resulted in a final monolayer edge line with a pronounced roughness dueto an inhomogeneous wetting of the polymer film (see Figure 4). Monolayers were obtained by spreading a lipid solution (chloroform, $1 \mathrm{mg} / \mathrm{mL}$; all solvents used in the present work were p.a. quality) on the trough subphase. After thelipid solution was spread the solvent was allowed to evaporate (for a $1 / 4 \mathrm{~h}$ ) and the film was compressed to the desired lateral pressure $\left(\mathrm{T}=25^{\circ} \mathrm{C}\right.$ ). After completion of compression the film was deposited without further delay (at a speed of $4 \mathrm{~mm} / \mathrm{min}$ ) toavoid significant spreading of themonolayer ontothepolymer support. To allow for visualization of the deposited monolayer by fluorescence microscopy, the lipid solution was doped by the addition of $1 \mathrm{~mol} \%$ of a lipid fluorescence probe. Prior to mounting of the monolayer-covered glass substrates into the humidity chamber, lipid transferred to the backside of the substrate was thoroughly wiped off with a 2-propanol-soaked tissue.

Lipids. The lipids DMPC (1,2-dimyristoyl-sn-glycero-3-phosphatidylcholine), DSPC (1,2-distearoyl-sn-glycero-3-phosphatidylcholine), and cholesterol (3 $\beta$-hydroxy-5-cholesten) were purchased from Avanti Polar Lipids, Alabaster, AL, and used without purification (the purity level was $>99 \%$, as stated by the manufacturer). The fluorescence probe used for staining the lipid monolayers was N-(7-nitrobenz-2-oxa-1,3-diazol-4-yl)-1,2dihexadecanoyl-sn-glycero-3-phosphoethanolamine, triethyl-

(26) Wolff, O. Ph.D. Thesis, Ruprecht-Karls-Universität, Heidel berg, Germany, 1998. 
ammonium salt (NBD-PE; Molecular Probes, Leiden, The Netherlands). I t was added to theli pid sol ution at a concentration of $1 \mathrm{~mol} \%$ with respect to the host lipid.

Polymer Cushions. Chitosan and agarosewereobtained from Fluka/Sigma Aldrich, Seelze, Germany, and used without purification. Chitosan was dissolved in a $1 \% \mathrm{v} / \mathrm{v}$ acetic acid solution (99.8\%; Riedel-de Haën, Seelze) ${ }^{27}$ at a concentration of $1 \% \mathrm{w} / \mathrm{w}$ with stirring overnight. The solutions were filtered through syringe filters (Millex, Millipore Corp., Bedford, MA) with a poresizeof $5 \mu \mathrm{m}$. Afterward the sol utions werecentrifuged (Biofuge22R, Heraeus, Germany) for $30 \mathrm{~min}$ at a speed of 11400 rpm. Thin chitosan films were prepared by spin-coating the chitosan solution onto cleaned, hydrophilic glass substrates. Substrate cleaning was performed by sonication in a $2 \% \mathrm{v} / \mathrm{v}$ Hellmanex solution (Hellma, Germany), followed by thoroughly rinsing the substrates in Millipore water. Spin-coating was typically performed at a spinning speed of $3000 \mathrm{rpm}$. Freshly prepared hydrogel layers were allowed to dry in air, for a period of typically $1 / 2 \mathrm{~h}$. Ellipsometry measurements revealed film thicknesses around $140 \mathrm{~nm}$. Toneutralizethefilms, the polymercovered substrates were immersed for several hours in a borate buffer (pH 9.22; Merck, Darmstadt, Germany) and afterward rinsed in Millipore water.

Agarose films were prepared by dipping dean substrates into a hot agarose solution of concentration $0.2 \% \mathrm{w} / \mathrm{w}$. U pon quick withdrawal, a thin polymer film remained on thegl ass and formed a thin gel film during cooling. ${ }^{28}$

\section{Theoretical Background of the Dynamics of Spreading Processes}

The spontaneous spreading of liquids on solid surfaces is controlled by the competition between driving terms and dissipative processes. ${ }^{29}$

The dynamics of a macroscopic droplet spreading on a solid can often be described by a relation known as the Tanner law. ${ }^{30}$ From that law it follows that the velocity of the spreading droplet front is essentially independent of the spreading power $\mathrm{S}$, which is the driving term for droplet spreading:

$$
\mathrm{S}=\gamma_{\mathrm{S}}-\gamma_{\mathrm{L}}-\gamma_{\mathrm{SL}}
$$

with $\gamma_{S}, \gamma_{L}$, and $\gamma_{S L}$ being the interfacial tensions of the solid and liquid surfaces and the solid/liquid interface, respectively.

However, it has long been known that the spreading of a macroscopic liquid droplet on a solid is in many cases preceded by a very thin, invisibl efilm, ${ }^{31}$ which occurs even when condensation from a vapor phasecan beneglected. ${ }^{32}$ Whereas the shape of the macroscopic droplet is a thermodynamic, macroscopic property, ${ }^{33}$ the surface profile of the precursor filmis controlled by long-rangesurface forces (between approximately $30 \AA$ and $1 \mu \mathrm{m}^{30}$ ). Where evaporation can be neglected, the thickness of the "completely" spread droplet is above the value of a monolayer, even in the case of a zero (macroscopic) contact angle. Liquids with large S, however, spread more efficiently than liquids of small $\mathrm{S}$. The independence of droplet spreading velocity on the spreading power can be explained by completeconsumption of the free energy (per unit area), S, through dissipative processes in the precur-

(27) Blair, H. S.; Huthrie, J .; Law, T.-K.; Turkington, P. J . Appl. Polym. Sci. 1987, 33, 641-656. 191.

(28) Dietrich, C.; Tampe, R. Biochim. Biophys. Acta 1995, 1238, 183-

(29) Valignat, M. P.; Voue, M.; Oshanin, G.; Cazabt, A. M. Colloids Surf., A: Physicochem. Eng. Aspects 1999, 154, 25-31.

(30) de Gennes, P. G. Rev. Mod. Phys. 1985, 57, 827-863.

(31) Hardy, W. Philos. Mag. 1919, 38, 49.

(32) Bangham, D.; Saweris, S. Trans. Faraday Soc. 1938, 33, 554

(33) I sraelachvili, J. N. Intermol ecular and SurfaceF orces, 2nd ed.; AcademicPress: London, San Diego, New Y ork, Boston, Sydney, Tokyo, Toronto, 1992. sor film. ${ }^{30}$ The spreading of films with mesoscopic thickness $^{29}$ can be described by continuum theories in terms of both statics (DLVO theory) and dynamics (hydrodynamics, Navier-Stokes equations).

Wetting of molecularly thin liquid films, however, is often accompanied by structuring effects. In fact, the films advance as a series of distinct mol ecular layers, ${ }^{34}$ or-in later spreading stages-as a singl e monomolecular layer. While for macroscopic and mesoscopic films a no-slip boundary at the solid/liquid interface applies (the dissi pation occurs in the bulk liquid, quantified by means of the viscosity $\eta$ ), in the case of molecularly thin layers dissipation occurs by friction at the solid support, on the molecular scale.

Themonolayer growth of a si mpleliquid, extending from a straight reservoir or from a droplet of radius $R_{0}$, has been extensively studied experimentally, mainly by means of spatially resolved ellipsometry, ${ }^{29,35-39}$ in combination with $A F M, 40$ or by spatially resolved surface plasmon spectroscopy. ${ }^{23} \mathrm{~N}$ umerous theoretical investigations have been carried out. ${ }^{41-45} \mathrm{I}$ n parallel, computer simulations ${ }^{46}$ have been performed.

In most of the above-mentioned works monolayer spreading was found tofoll low a square-root behavior with respect to the timedependence of thespreading distance. In particular, the model developed in refs 43-45 leads to the following equation for a monolayer spreading from a bulk droplet with radius $\mathrm{R}_{0}$ :

$$
R(t)=R_{0}+A\left(D_{0} t\right)^{1 / 2}
$$

In eq 2, A is a parameter which may be positive (wetting), negative(dewetting), or zeroand depends on temperature and particle/particle and particle/substrate interactions. $D_{0}$ is the diffusion coefficient of an i sol ated liquid molecule on the surface. I nterestingly, A depends mainly on liquid/ liquid interactions and is almost independent of the spreading power. ${ }^{44}$

Apart from pure simple liquids, wetting of solids often occurs by or in the presence of surface-active compounds such as surfactants and lipid molecules. Wetting by amphi philes is highly facilitated in the presence of water films ranging in thickness from the molecular to the macroscopic scale. The kinetics of spreading in these cases are accompanied by additional phenomena such as superspreading, ${ }^{47}$ fingering instabilities, ${ }^{48-50}$ and sol itary waves. ${ }^{51-53}$ 642.

(34) Heslot, F.; Fraysse, N.; Cazabat, A. M. Nature 1989, 338, 640-

(35) Heslot, F.; Cazabat, A. M.; Levinson, P. Phys. Rev. Lett. 1988, $62,1286-1289$.

(36) Fraysse, N.; Valignat, M.P.; Cazabat, A. M.; Heslot, F.; Levinsson, P. J. Colloid Interface Sci. 1993, 158, 27-32.

(37) Cazabat, A. M.; De Coninck, J .; Hoorel beke, S.; Valignat, M. P.; Villette, S. Phys. Rev. E 1994, 49, 4149-4153.

(38) Voue, M.; Valignat, M. P.; Oshanin, G.; Cazabat, A. M. Langmuir 1999, 15, 1522-1527.

(39) Blake, T. D.; Decamps, C.; De Connink, J .; de Ruijter, M.; Voue, M. Colloids Surf., A: Physicochem. Eng. Aspects 1999, 154, 5-11.

(40) Villette, S.; Valignat, M. P.; Cazabat, A. M.; Schabert, F. A Kalachev, A. Physica A 1997, 236, 123-129.

(41) Abraham, D. B.; Collet, P.; Coninck, J . D.; Dunlop, F. Phys. Rev. Lett. 1990, 65, 195-198.

(42) Coninck, J . D.; Dunlop, F.; Menu, F. Phys. Rev. E 1993, 47 $1820-1823$

(43) Burlatsky, S. F.; Oshanin, G.; Cazabat, A.-M.; Moreau, M. Phys. Rev. Lett. 1996, 76, 86-89.

(44) Burlatsky, S. F.; Oshanin, G.; Cazabat, A.-M.; Moreau, M.; Reinhardt, W. P. Phys. Rev. E 1996, 54, 3832-3845.

(45) Oshanin, G.; De Coninck, J . D.; Cazabat, A. M.; Moreau, M. J. Mol. Liq. 1998, 76, 195-219.

(46) De Coninck, J .; Fraysse, N.; Valignat, M. P.; Cazabat, A. M Langmuir 1993, 9, 1906-1909.

(47) Hill, R. M. Curr. Opin. Colloid Interface Sci. 1998, 3, 247-254. 

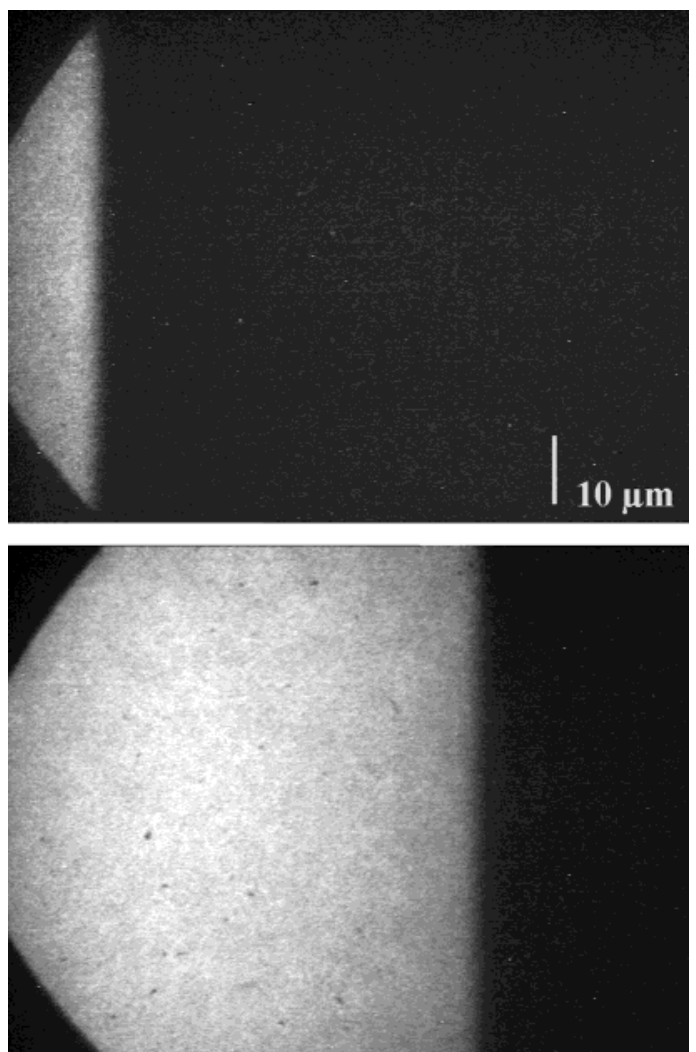

Figure 2. DMPC monolayer on a chitosan film, lateral pressure $50 \mathrm{mN} / \mathrm{m}$, relative humidity $90 \%$ : top, beginning of the spreading experiment; bottom, 9 min later. Note: the broadening of the spreading front during the spreading process was negligibly small.

The spreading dynamics of amphiphile monolayers on water films can becomplex, and the form of the spreading law depends on the film thickness and monolayer geometry as well as on the ratio of the Marangoni driving term (surface tension gradient) to the diffusion coefficient; see eq 4 . In the case of a constant film thickness, a constant spreading velocity proportional to the surface pressure gradient and the film thickness was found, ${ }^{54}$ accor ding to

$$
\frac{\Delta \pi}{\mathrm{l}}=\frac{\eta_{\mathrm{w}} \mathrm{V}}{\mathrm{d}}
$$

where $\pi$ is the surface pressure, $I$ is the length of the spreading film, $\eta_{\mathrm{w}}$ is the water viscosity, vis thespreading velocity, and $d$ is the water film thickness.

\section{Results}

Thelateral expansion and spreading of lipid monolayers onto uncovered substrate areas was observed by fluorescence mi croscopy at increased relativehumidities. At room humidity ( $\mathrm{RH} \approx 50 \%$ ), however, the spreading velocity was too small to be detectable. Observation of the monolayer at room humidity right after the LB transfer revealed in most cases a sharp monolayer edge (Figure2,

(48) Troian, S. M.; Wu, X. L.; Safran, S. A. Phys. Rev. Lett. 1989, 62, 1496-1499.

(49) Troian, S. M.; Herbolzheimer, E.; Safran, S. A. Phys. Rev. Lett. 1990, 65, 333-336.

(50) Elender, G.; Sackmann, E. J . Phys. II 1994, 4, 455-479.

(51) Borgas, M. S.; Grotberg, J . B. J . Fluid Mech. 1988, 193, 151170.

(52) J ensen, O. E.; Grottberg, J . B. J . Fluid Mech. 1992, 240, 259288.

(53) Frey, W.; Sackmann, E. Langmuir 1992, 8, 3150-3154.

(54) He, S.; Ketterson, J . B. Philos. Mag. B 1998, 77, 831-847. top). Only in cases where the lipid solution was deposited rapidly after the substrate was immersed into the LB trough a rough monolayer edge was observed (F igure 4), whereas equilibrating the substrate with the subphase prior to the deposition of lipid solution led to smooth monolayer edges. From the fluorescence intensity profile past the initial monolayer edge prior to performing a spreading experiment (Figure 3), it can be deduced that the edge width (about $1 \mu \mathrm{m}$ ) was in the range of the resolution of the optical microscope.

Spreading of DMPC on Chitosan Surfaces. Increasing the relative humidity to a value of $\mathrm{RH}=90 \%$ substantially increased the monolayer mobility, which resulted in a movement of the spreading front (Figure 2, bottom). The kinetics of thespreading front displacement could be reversibly changed by altering $\mathrm{RH}$, which indicates that spreading velocities were controlled by the water content of the surrounding gas phase, and not by theresidual water content of the hydrogel layer stemming from immersion into the water subphase during the Langmuir-Blodgett transfer process. Accompanying the monolayer edge displacement, a slight broadening of the edgewidth occurred, as deduced from theintensity profiles shown in Figure 3. The intensity profiles were obtained by evaluating gray levels of pixels in a box-shaped region of interest and integrating along the direction of the monolayer edge line. The subtle broadening could be due to either a slight increase of the edgeroughness or a small density gradient induced by spreading. Additionally, the reduced fluorescence intensity at the monol ayer edge could be due to a higher friction of the fluorescence probe with the underlying support, compared to the unlabeled lipid molecules. Besides the slight intensity loss at the monolayer edge, thefluorescence intensity of thenewly covered substrate area corresponded to the intensity observed in the initially covered area (see Figures 2 and 4).

As has been mentioned already, insufficient equilibration of the polymer cushion in the aqueous subphase led to a curvy monolayer edge. However, during the spreading process at an increased $\mathrm{RH}$ the edgestructuring leveled out (Figure 4) while spreading over a distance of less than $100 \mu \mathrm{m}$. Also in Figure 4, it can clearly be seen that the intensity of the spreading monolayer is constant before and after spreading over a distance of dozens of micrometers.

Spreading experiments for time-dependent quantitative measurements were performed with samples where the spreading front appeared smooth right from the start. Monolayersweretransferred at different lateral pressures, and the displacement of the spreading front relative to thestarting position was determined at different spreading times. Figure 5 shows a typical development of the spreading front. In that experiment, a DM PC monolayer was transferred at a lateral pressure of $20 \mathrm{mN} / \mathrm{m}$. The position of the monolayer edge was determined, and afterward, $\mathrm{RH}$ was set to $90 \%$. An almost perfectly linear relation between spreading front displacement and time (F igure 5) was found for all lateral pressures, $\pi$, and RHs. The final spreading distance was in all cases below 90 $\mu \mathrm{m}$, which is small compared to the initial total length $(1.8 \mathrm{~cm})$ of the monolayer. Hence, the monolayer expands only by a factor of $<0.5 \%$ and ther efore can be regarded as an infinite lipid reservoir.

Figure6 shows theinfluence of thelateral film pressure (taken as the initial film pressure adjusted on the Langmuir-Blodgett trough during monolayer deposition) on spreading vel ociti es of chitosan-supported monolayers at two different relative humidities ( $\mathrm{RH}=85 \%$ and $90 \%$ ). Theerror bars werecal culated from theerror of linear fits 


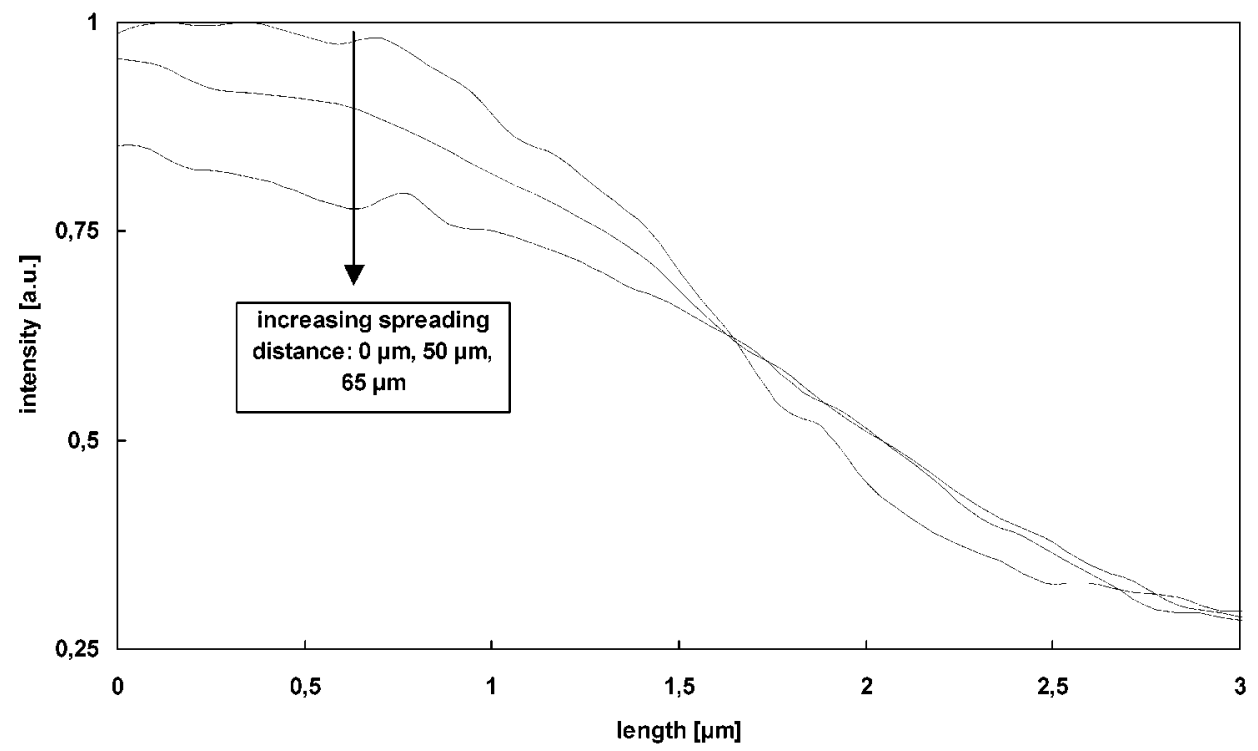

Figure 3. Intensity profiles of the monolayer edge (along a line orthogonal to the edge) during spreading. Note: the width of the edge prior to spreading is comparable to the resolution of the optical microscope. The intensity profiles have been shifted arbitrarily with respect to the length axis. Profiles were examined directly after deposition $(0 \mu \mathrm{m})$, and after spreading over distances of 50 and $65 \mu \mathrm{m}$.
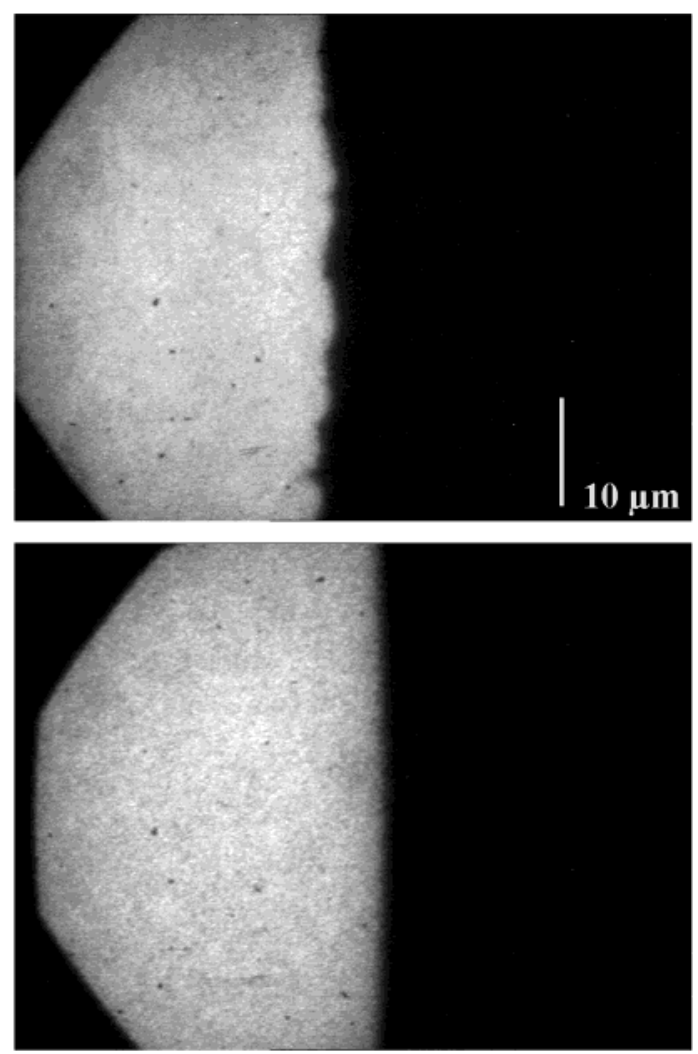

Figure4. DMPC monolayer on a chitosan film, lateral pressure $20 \mathrm{mN} / \mathrm{m}$, relative humidity $90 \%$ : top, prior to increasing humidity; bottom, 50 min later. In the case of the top picture, the sample had been shifted to compensate for the movement of the spreading front (approximately $40 \mu \mathrm{m}$ ).

to the data series (Figure 5) and do not include error estimates from repeated measurements.

From the diagram, the following conclusions can be drawn: (I)RH showed a stronginfluenceon the spreading vel ocity. In the case of a DM PC monolayer with a lateral pressure of $50 \mathrm{mN} / \mathrm{m}$, a change of $\mathrm{RH}$ from $85 \%$ to $90 \%$ led to an increase of the spreading vel ocity from 5.3 to 25 $\mu \mathrm{m} / \mathrm{min}$, i.e., a factor of 4.7 . (II) The lateral pressure set on the LB trough during monolayer deposition strongly

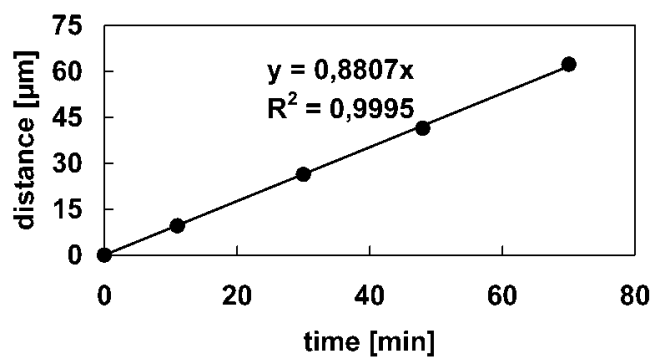

Figure 5. Spreading front displ acement of a DMPC monolayer on chitosan, lateral pressure $20 \mathrm{mN} / \mathrm{m}$, relative humidity $90 \%$. The slope of a linear fit yielded a velocity of $0.88 \mu \mathrm{m} / \mathrm{min}$, corresponding to $0.015 \mu \mathrm{m} / \mathrm{s}$.

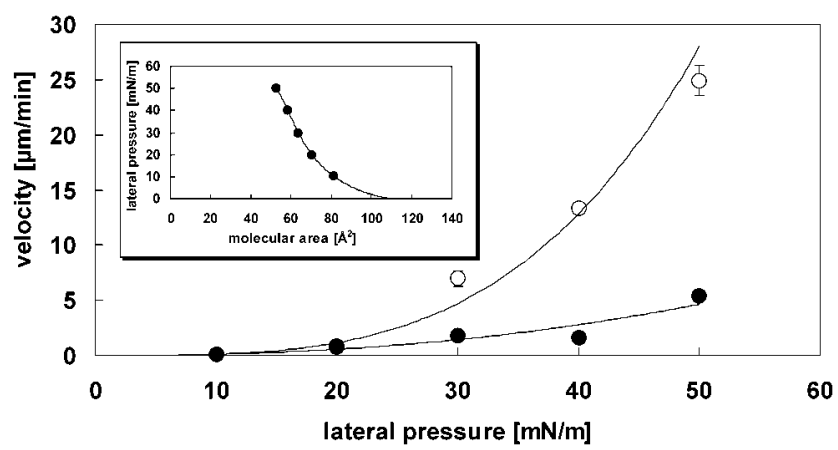

Figure 6. Comparison of the spreading velocities of DMPC monolayers transferred at different lateral pressures at two different relative humidities: closed circles: $\mathrm{RH}=85 \%$; open circles, $\mathrm{RH}=90 \%$. The lines are guides to the eyes. Inset: pressure/area isotherm of DMPC at $25^{\circ} \mathrm{C}$; the dots refer to the transfer pressures.

influenced the spreading velocity. At a relative humidity of $90 \%$, a change from $\pi=10 \mathrm{mN} / \mathrm{m}$ to $\pi=50 \mathrm{mN} / \mathrm{m}$ increased the spreading velocity from 0.1 to $25 \mu \mathrm{m} / \mathrm{min}$, i.e., by a factor of 250. (III) The most interesting finding is that the relation between spreading vel ocity and lateral monolayer pressure $\pi$ was nonl inear, which is contrary to spreading of lipid on a water film of mesoscopicthickness. ${ }^{54}$ Instead, the sl ope of an arbitrary fit to the data increases monotonically (Figure 5). At a lateral pressure of $50 \mathrm{mN} / \mathrm{m}$ at $25^{\circ} \mathrm{C}$, theDM PC monolayer was just in the coexistence 

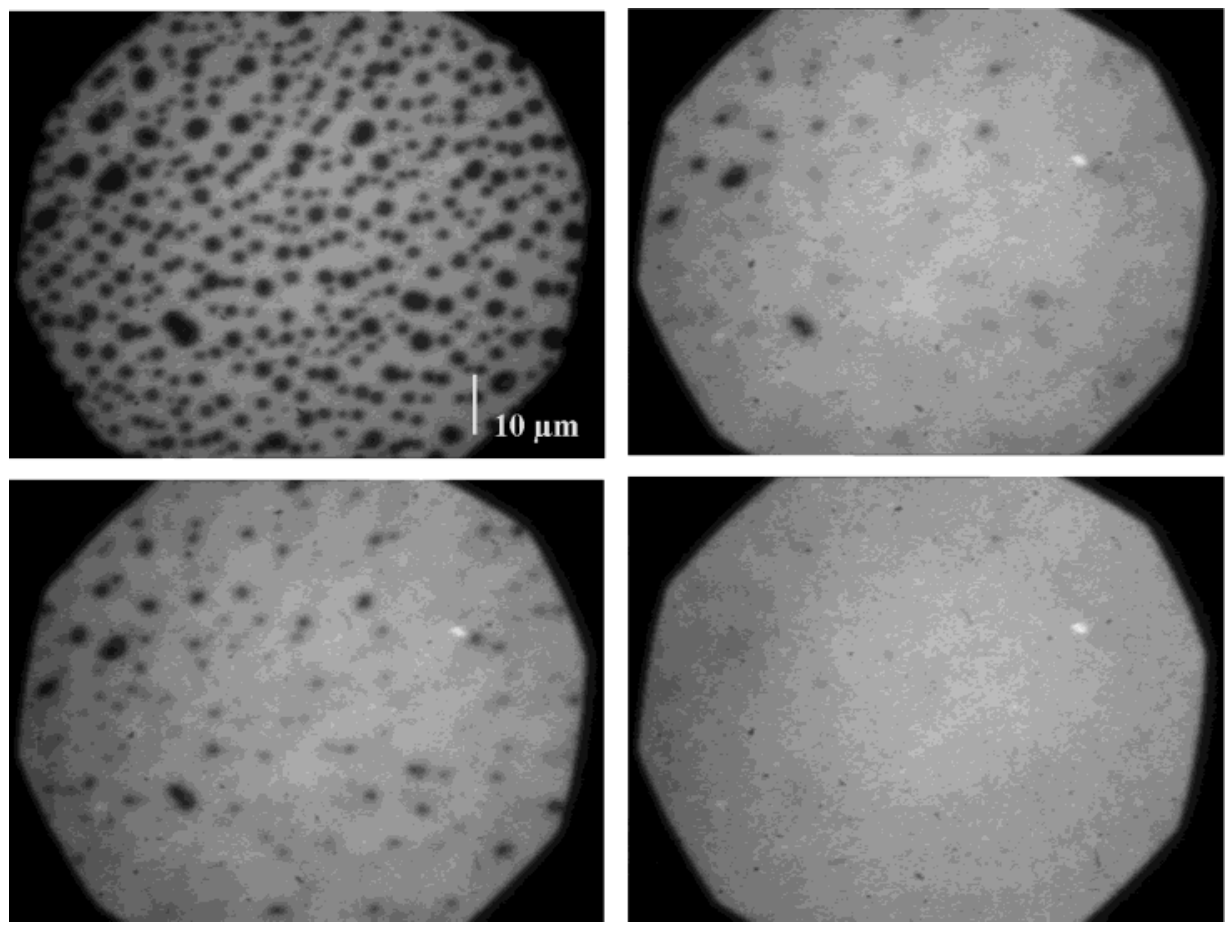

Figure 7. Fluorescenceimages showing(A, top left) thestructure of a lipid monolayer (DMPC, lateral pressure $35 \mathrm{mN} / \mathrm{m}$, fluorescence probe NBD-PC) which had been subjected to a condensation of water droplets, and which had been dried rapidly afterward, and ( $B$, bottom left; $C$, top right; $D$, bottom right) the same location on the same monolayer as in ( $A$ ), after switching to a high (near condensation) humidity. The time interval between each picture was approximately 1 min.

region, which, however, did not seem to influence the spreading velocity.

Figure 7 shows that monolayers resting on a hydrated polymer cushion at an increased relativehumidity possess a self-healing property. Defects, which were caused by a condensation of water droplets on the monolayer surface due to a temperature gradi ent across the gl ass substrate, could befixed in themonolayer by rapid dehydration. The condensation and evaporation of water droplets was followed by means of reflection interference contrast microscopy ( $\mathrm{RICM}^{24}$ ). I nterference patterns generated by this approach revealed a complete evaporation of water droplets during the dehydration step. Changing $\mathrm{RH}$ to a value just bel ow the dew point caused a recovery of the initial homogeneous monolayer structure, i.e., a selfrepairing by the lateral spreading of lipid into the monolayer holes.

Spreading of DSPC and DMPC/Cholesterol Mixtures on Chitosan. To analyzetheinfluence of thephase state on the spreading behavior, a DSPC monolayer was transferred to a chitosan cushion at a lateral pressure of $40 \mathrm{mN} / \mathrm{m}$, where DSPC is completely in the liquid condensed state. Figure8 shows that, contrary to thecase of a fluid monolayer (Figure 2), a relatively diffuse monolayer edge was obtained. Moreover, the film was rather inhomogeneous. It showed hol es and cracks, which stem from the brittlecondition of a condensed monolayer, in combination with a highly reduced mobility. In fact, raising $\mathrm{RH}$ even to values cl ose to the dew point could not induceany observablespreading during thetime of several hours.

Furthermore, theinfluenceof thepresence of chol esterol was examined. A DMPC monolayer with an additional amount of $30 \mathrm{~mol} \%$ cholesterol was transferred onto a chitosan cushion at a lateral pressure of $40 \mathrm{mN} / \mathrm{m}$ at 25 ${ }^{\circ} \mathrm{C}$. A spreading vel ocity of $0.16 \pm 0.009 \mu \mathrm{m} / \mathrm{min}$ was found at $\mathrm{RH}=90 \%$. Thus, the spreading velocity was reduced considerably compared to thevaluefound for a pureDM PC

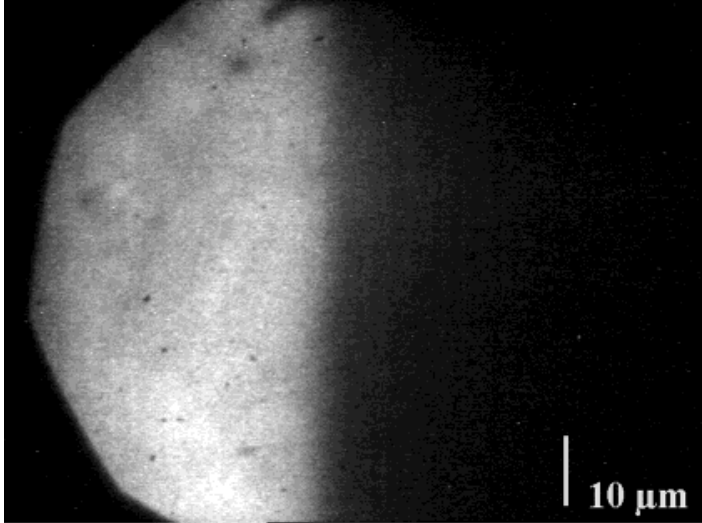

Figure 8. Monolayer edge of DSPC on chitosan, lateral transfer pressure $40 \mathrm{mN} / \mathrm{m}$.

monolayer, transferred at the same conditions (spreading velocity at $40 \mathrm{mN} / \mathrm{m}$ and $\mathrm{RH}=90 \%, 13.4 \pm 0.37 \mu \mathrm{m} / \mathrm{min}$ ). The influence of cholesterol on supported membrane dynamics is manifold. Cholesterol reduces the diffusion coefficients measured in phospholipid membranes considerably. ${ }^{55}$ Additionally, the presence of chol esterol leads to a dehydration of the membrane substrate gap, ${ }^{25}$ which results in a further decrease in spreading vel ocity.

Spreading of DMPC on Different Substrates: Agarose and Glass. To demonstrate theinfluence of the nature of themonolayer substrate on spreading vel ocities, a DM PC monolayer with a lateral transfer pressure of 40 $\mathrm{mN} / \mathrm{m}$ was deposited onto agarose and in a different experiment directly onto glass. In the case of the former, the spreading velocity at $\mathrm{RH}=90 \%$ was $1.00 \pm 0.020$ $\mu \mathrm{m} / \mathrm{min}$, hence considerably smaller as compared to that of a DM PC monolayer on chitosan prepared and analyzed under the same conditions (see above). One reason for

(55) Almeida, P. F . F .; Vaz, W. L. C.; Thompson, T. E. Biochemistry 1992, 31, 6739-6741. 
such a decrease of thespreading vel ocity is the pronounced roughness of agarose in comparison to chitosan. Additionally, it could bethe case that the swelling behaviors of the polymer cushions chitosan and agarose are different.

On glass, the situation for a DMPC monolayer, lateral pressure of $40 \mathrm{mN} / \mathrm{m}$ at $25^{\circ} \mathrm{C}$ and relative humidity of $90 \%$, was similar tothat of a DSPC monolayer on chitosan, exposed to the same conditions. On one hand, a clearly visi ble boundary between covered and uncovered substrate surfaces was observed (fluorescence picture not shown). On the other hand, nodisplacement of the spreading front was observed during several hours of exposure to extremely high relative humidities.

\section{Discussion}

As indicated in Figures 2 and 4, a sharp boundary (at the resolution of the optical microscope) between monolayer-covered and uncover ed substrates was obtained right after the LB transfer. In cases where insufficient equilibration of polymer-covered substrates in the LB trough caused a curvy three-phase line, the initially structured monolayer edge leveled out during spreading to yield an essentially straight line while spreading over a distance smaller than $100 \mu \mathrm{m}$. Furthermore, the monolayer edge width, which was below the resolution of the optical microscope before spreading, slightly increased during the spreading process. Moreover, the monolayer edge displacement velocity was strongly dependent on the lateral compression of the monolayer on the LB trough before transfer. These observations indicate that (I) spreading of the monolayer before the completion of the LB transfer, i.e., while the substrate was still immersed in the LB trough, can be neglected, (II) the position of the monolayer edge before spreading is determined by the position of the three-phase line in the LB trough before addition of lipid to the subphase surface, and (III) the lipid density at the monolayer edge is determined by the lateral pressure of thelipid monolayer on the LB trough.

The development of the meniscus at the three-phase lineafter addition of lipid solution tothesubphasesurface (before compression) was analyzed by Yaminsky et al. ${ }^{56}$ These authors found that the three-phase line at a mica substrate, half immersed into a pure water subphase, retracts several hundreds of micrometers upon the addition of lipid solution (DSPE) onto the subphase. Upon retraction, the contact angle increased due to the deposition of lipid onto the solid, at a pressure of several $\mathrm{mN} / \mathrm{m}$, although the lateral pressure within the monolayer on the subphase was still bel ow $0.1 \mathrm{mN} / \mathrm{m}$. Thecontact angle (and therefore the lateral pressure of the monolayer deposited during meniscus retraction) depended on the amount of lipid solution initial ly loaded ontothesubphase. The pressure difference between a monolayer deposited onto a substrate and that remaining on the LB trough subphase depends on the strength of the headgroup/ substrate interaction. ${ }^{56}$ As in the present work in most cases a hydrogel was used as a substrate, it can beassumed that theretraction of theinitial three-phaselineis grossly reduced, if not diminished completely. This assumption is supported by theobservation that thespreading vel ocity did not depend on the amount of lipid solution added to thesubphasesurface, but on thelateral pressureobtained after compression of the monolayer on the LB trough.

The smooth spreading front of lipid monolayers is in contrast to the spreading front of phospholipid bilayers

(56) Yaminsky, V.; Nylander, T.; Ninham, B. Langmuir 1997, 13, 1746-1757

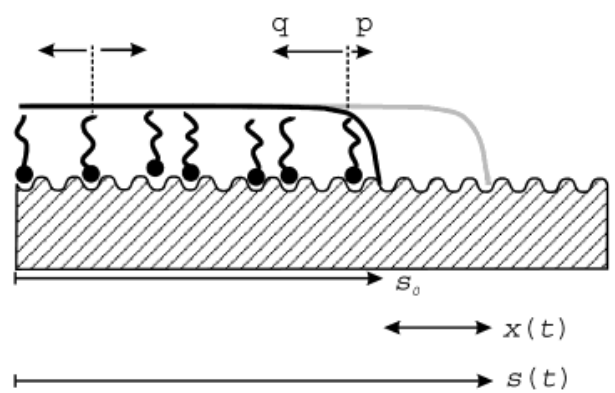

Figure 9. Spreading of a lipid monolayer initially confined to a fraction of the whol e substrate area. Molecules in the "bulk" film have the same transition probabilities in each direction. Boundary particles, however, have a higher tendency, $q>p$, tojump intothedirection of thefilm, duetolong-rangeattractive forces on the particle exerted by the monolayer. $\mathrm{s}_{0}=$ initial length of the monolayer film, $s(t)=$ total length of the film at time $t$, and $x(t)=$ spreading distance at time $t$. The polymer supporting the lipid monolayer has been omitted for clarity.

on hydrophilicsurfaces, wherea steady roughening during spreading was observed. ${ }^{14,24}$ The latter was explained by a percolation process: smooth spreading is obstructed by pinning centers at low spreading velocities. As the spreading coefficient in the case of monolayer spreading is much higher compared to that of bilayer spreading, ${ }^{17}$ percolation due to pinning centers is likely to be reduced considerably.

Analyzing the dynamics of monolayer spreading revealed a linear relationship between the spreading front displacement and the observation time (Figure 5). The spreading vel ocity was dependent on monolayer pressure and the relative humidity.

The physical analysis of the spreading of a quasi twodimensional layer on a solid can be reduced to a onedimensional problem, ${ }^{43}$ as depicted in Figure 9 . In refs 43 and 57, particle dynamics were modeled as an activated random hopping transport constrained by hard-core(shortrange) interactions between the local mi nima of potential wells in a square lattice (Figure 9). The wells are deep with respect to complete desorption to the gas phase, but act as a much lower barrier against the movement across the surface. Hops of diffusors only take place if the neighboring well is vacant. All particles, except those located at the film boundary, have isotropic transition rates: the probability to hop in any of the four directions is $1 / 4$. However, at the film boundary transition rates are different: the probability, q, for a boundary particle to hop in the direction of the film exceeds the probability, $p$, to movetoward thefreesurface. Advancement of the film, however, occurs because vacancies created by a boundary particle, while hopping toward the free surface, are filled by "bulk" monolayer partides. Theasymmetry of transition rates at the boundary arises because of long-range (van der Waals) attractions. Thus, the "bulk"monolayer exerts a force on boundary particles, which drags them toward the film, and monolayer spreading can be regarded as liquid-like.

The hopping model proposed in refs 43 and 44 for monolayer spreading froman infinitebulk-liquid reservoir and applied to the spreading of a semi-infinite monolayer in a refined form in refs 45 and 57 predicts a square root behavior of the edge displacement with respect to the observation time.

According to ref 19 , the ratio between advection-like spreading and spreading by diffusion is described by a

(57) Oshanin, G.; Coninck, J . D.; Cazabat, A. M.; Moreau, M. Phys. Rev. E 1998, 58, 20-23. 
surface Peclet number, which in the present case reads

$$
\mathrm{Pe}=\frac{\mathrm{W}_{\mathrm{A}}}{\xi \mathrm{D}}
$$

$W_{A}$ is the differencein surfaceenergies of the covered and uncovered areas, $\xi$ is a surface friction coefficient, and D is the diffusion coefficient of an isolated molecule. Note that the definition of the Peclet number is lent from hydrodynamics, and for the quantity $\xi$, the restrictions mentioned bel ow hold. In the case of high Peclet numbers spreading by advection is favored. Spreading by advection leads to a constant spreading velocity as long as the pressuregradient $\Delta \pi$ across thespreading front is constant (see eq 3).

Borgas and Grotberg ${ }^{51}$ theoretically predicted that the monolayer velocity on a thin water film is constant in the steady state (in the case of a constant monol ayer length). In refs 50 and 54, a constant spreading velocity for monolayer spreading on a thin (mesoscopic) water film was found experimentally. $\mathrm{He}$ and Ketterson ${ }^{54}$ studied therate of lipid exchange between monolayers at differ ent lateral pressures on two LB troughs, connected by a glass bridge covered by a thin water film. A linear dependence of monolayer velocity on the surface pressure difference, $\Delta \pi$, was found (eq 3 ) in the case of low velocities.

In the following, the time dependence of monolayer spreading will be analyzed by adapting a model which was proposed in ref 24 for the analysis of the spreading of a lipid bilayer on a solid substrate from an infinite reservoir. In ref 24, a stationary equilibrium is assumed; i.e., the gain in free energy per area, $W_{A}$, by bilayer spreading is equal to the energy dissipation by friction:

$$
W_{A} d A=(f d y) d s
$$

where $d A=d y d s$ is the area covered by the spreading film element ( $y$ is the width of the proceeding rim) and $f$ is the viscous force per unit length of the proceeding rim. Further, a linear dependence of $f$ on sliding vel ocity, $v(s, t)$ $=\mathrm{ds} / \mathrm{dt}$, as well as on the total length of the bilayer film is assumed:

$$
f=\xi \frac{d s(t)}{d t} s(t)
$$

Combining eqs 5 and 6 then leads after integration to the spreading law

$$
v(t)=\left(\frac{W_{A}}{2 \xi}\right)^{1 / 2} \frac{1}{t^{1 / 2}}
$$

and hence to $\mathrm{t}^{-1 / 2}$ dependence of the spreading vel ocity. $\xi$ is a viscous drag coefficient ${ }^{24}$ which was interpreted in terms of a linear velocity gradient in the water film between the solid support and lipid membrane. Assuming no-slip boundaries at the membrane and solid surface yields $\xi=\eta_{\mathrm{w}} / \mathrm{d}$, where $\eta_{\mathrm{w}}$ is the bulk water viscosity and $d$ the thickness of the water film. F urthermore, dehydration of the membrane/substrate gap due to the presence of moderate amounts of cholesterol was found to lower bilayer spreading significantly. ${ }^{25}$ In that case, $\xi$ was interpreted as describing the friction between two monolayers, the first one (proximal layer) being fixed and the second one (distal layer) sliding over the first one. The meaning of $\xi$ in the case of films with a thickness in the molecular range, however, is not obvious. In fact, what friction means at the molecular scale and which parameters are relevant is currently still a matter of debate. ${ }^{29}$
In the following, a model will be derived which follows closely the argumentation provided in ref 24 . The main difference lies in the fact that, in ref 24 , the spreading reservoir was a hydrated bilayer stack, whereas in the present case, the spreading reservoir is the monolayer itself. In the case of small spreading distances $\left(\mathrm{x}(\mathrm{t}) \ll \mathrm{s}_{0}\right.$; see Figure 9), the monol ayer acts as an infinite reservoir. In the configuration depicted in Figure 9, the monolayer is initially confined to an area defined by length $\mathrm{s}_{0}$ (and width y). Hence, the viscous force per unit length equation yields

$$
f=\xi \frac{d s(t)}{d t} s(t)=\xi \frac{d s(t)}{d t}\left(s_{0}+x(t)\right)
$$

where the monolayer is assumed to behave rheologically as a Newtonian interface, ${ }^{58}$ which is also assumed to be the case for direct friction with a solid support. ${ }^{59} \mathrm{Ad}$ ditionally, it is supposed that dissi pation occurs in a region with a length $\mathrm{s}_{0}$, which is large compared to the spreading region $x(t)$. Whether $s_{0}$ refers tothewholeinitial monolayer length or to a region smaller than the whole monolayer, but much larger than the spreading front displacement $x(t)$ can in principle be proved by examining monolayer films with different initial lengths, which, however, was not carried out in the present work.

The parameter $\xi$ in the present experimental configuration quantifies the viscous friction at the monolayer/ substrateinterface. Possibly, theremight bean additional contribution of the dilational viscosity of the monolayer. ${ }^{51,60,61}$ Combination of eqs 8 and 5 yields

$$
\left(s_{0}+x(t)\right) d s(t)=\left(s_{0}+x(t)\right) d x(t)=\frac{W_{A}}{\zeta} d t
$$

and after integration

$$
\mathrm{s}_{0} \mathrm{x}(\mathrm{t})+\frac{1}{2} \mathrm{x}(\mathrm{t})^{2}=\frac{\mathrm{W}_{\mathrm{A}_{\mathrm{A}}}}{\zeta} \mathrm{t}
$$

With $s_{0} \gg x(t)$, eq 10 reduces finally to

$$
x(t)=\frac{W_{A}}{\xi \mathrm{s}_{0}} t
$$

From eq 11 it fol lows that thespreading front displacement is proportional to the energy per unit area $W_{A}$ gained by spreading, and inversely proportional to the friction coefficient $\xi$ and the length $\mathrm{s}_{0}$ of the region in which dissipation occurs. Possibly, so might correspond toa region with a smaller length than theinitial filmlength. However, this does not affect the derivation of eq 11 , as long as $\mathrm{s}_{0}$ is much larger than $\mathrm{x}(\mathrm{t}) . \mathrm{W}_{\mathrm{A}}$ is the difference in surface energy of the monolayer-covered and the monol ayer-free areas and may therefore be approximated by the monolayer film pressure $\pi$, which in the case of spreading of a monolayer onto alipid-freearea is equal tothedifference in film pressures $\Delta \pi$. The spreading described by eq 11 therefore is driven by a Marangoni force, i.e., a surface tension gradient.

Thus, for small spreading distances, $x(t)$, in contrast to eq 7, a constant velocity is obtained, as long as $W_{A} / \zeta$ is constant. Thelatter condition is fulfilled if the monolayer

(58) Scriven, L. E. Chem. Eng. Sci. 1960, 12, 98-108.

(59) Evans, E.; Sackmann, E. J . Fluid Mech. 1988, 194, 553-561.

(60) Hirsa, A.; K orenowski, G. M.; Logory, L. M.;j udd, C. D. Langmuir 1997, 13, 3813-3822.

(61) Wüstneck, R.; Wüstneck, N.; Grigoriev, D. O.; Pison, U.; Miller, R. Colloids Surf., B: Biointerfaces 1999, 15, 275-288. 
density reduction during spreading is small. The strong influence of the lateral pressure is in contrast to the diffusion model developed in ref 44 , where a negligible influence of the spreading power (which is proportional to the lateral monolayer pressure; see eq 1) on the rate of monolayer growth was found. Thus, besides theconstant velocity during spreading, the strong influence of the spreading power on the spreading vel ocity al so favors the model developed above (eq 11).

The fluorescence intensity distribution over the whole spreading distance did not change significantly during spreading, which is contrary to the diffusion model developed in ref 43 , where an s-shaped intensity profile was found in the spreading region, $x(t)$ (compare Figure 9). Ther efore, besides theconstant spreading vel ocity and the strong influence of monolayer pressure, the constant fluorescenceintensity in thespreading region (apart from a slight increase of the monolayer edge width) also supports theassumption that monolayer spreading in the present case is dominated by advection.

According to F igure6, thespreading vel ocity is strongly influenced by the relative humidity of the surrounding gas phase. For a given monolayer, $\mathrm{W}_{\mathrm{A}}$ should not depend remarkably on humidity; thus, theviscous drag coefficient $\xi$ is the valueresponsiblefor changing spreading vel ocities with varying humidities. A linear relationship of spreading velocity and lateral monolayer pressure (for $\mathrm{W}_{\mathrm{A}} / \xi \mathrm{s}_{0}=$ constant) was not found in the present experiments.

In all cases, monolayer dilation was below $0.5 \%$ of its original density. Hence, the influence of a shrinking monolayer reservoir can be neglected and therefore $\mathrm{W}_{\mathrm{A}}$ assumed to be constant. Theobserved nonlinearity can be explained by means of eq 11 by assuming that $\xi$ depends on thelateral fil $m$ pressure. Onecould arguethat a change in headgroup density is accompanied by a change in the friction coefficient $\zeta$. In fact a hydrodynamic relation between $\xi$ and the self-diffusion coefficient exists. ${ }^{16,25,59}$ The self-diffusion coefficient, however, increases with decreasing monolayer density. ${ }^{62}$ This effect would accelerate spreading at lower lateral pressures, which is not consistent with the data.

The influence of $\mathrm{RH}$ on $\xi$ is possibly due to a change of themonolayer/substratedisjoining pressure, which in the present case is dominated by hydration forces. ${ }^{33}$ It could be the case that the disjoining pressure depends not only on $\mathrm{RH}$ but al so on $\pi$. In thecase of protrusion forces causing the hydration force, ${ }^{63}$ the prefactor of the exponential relation between disjoining pressure and separation distance depends on the protrusion sitedensity. A reduced lateral pressureleads toa reduced protrusion sitedensity, which could possibly result in a smaller hydration force, leading to increased friction in the case of monolayers with small lateral pressures.

In principle, the drag coefficient $\xi$ can be determined by diffusion measurements and compared to those found from spreading experiments. ${ }^{25}$ In the present case, however, the contribution of the dilational viscosity of the lipid monolayer to the dissipation is unknown. Furthermore, to beabletocalculate $\xi$ from spreading experiments, the length of the dissipation region must be known. Therefore, a comparison of friction coefficients obtained from spreading and diffusion experiments was not carried out.

Thespreading vel ocity changed drastical ly whileeither the kind of monolayer support or the phase state of the

(62) Peters, R.; Beck, K. Proc. Natl . Acad. Sci. U.S.A. 1983, 80, 71837187. 876. deposited monolayer was varied. In the case of DSPC spreading on chitosan, according to eq 11, the friction coefficient $\xi$ is highly increased. Thus, the magnitude of friction on the solid support is connected to the lateral fluidity of the monolayer membrane, which in the solid and in the fluid state can differ by several orders of magnitude. ${ }^{62}$

\section{Conclusion}

A self-repairing ability of physisorbed amphiphile monolayers on hydrophilic substrates was observed and quantitatively analyzed by covering half of a planar hydrogel surface with a Langmuir-Blodgett film, raising the relative humidity considerably and examining the spreading kinetics of the monolayer onto the uncovered area. Directly after the transfer a very sharp and linear monolayer edge was observed, provided the hydrogel was equil ibrated sufficiently with thetrough subphase. In the case of film edge distortions being observed right after thetransfer, theseheal ed out quickly during thespreading process toyield a straight and sharp monolayer edge. This is in contrast to bilayer spreading, which was explained by the much higher spreading power in the case of monolayer spreading. A spreading front displacement linear with time was found for a lateral expansion of the monolayer of less than $0.5 \%$. This was explained by assuming an expansion over a large film area and balancing the resulting dissipation by friction at the monolayer substrate interface with the driving force, the high surface energy of a free hydrogel surface. A simple model was derived which describes the dependence of the spreading velocity on the system parameters. The following features were found to influence the spreading dynamics: (I) thetransfer pressure of the monolayer, (II) the relative humidity, (III) the phase state of the monolayer, (IV) the cholesterol content of the monolayer, (V) the nature of the substrate.

The dependence of the spreading vel ocity on thedriving force was found to be nonlinear, in contrast to the spreading velocities obtained on a thin water film sublayer, ${ }^{54}$ which could be explained by assuming that the friction at the monolayer/substrate interface varies with the monolayer density.

It is important tonotethat a retraction of themonolayer leading edge while the val ue of $\mathrm{RH}$ is reduced was never observed. In fact, in multibilayer stacks changing RH leads to an additional effective lateral pressure, ${ }^{64}$ which compresses the bilayer and can therefore induce phase transitions (see, e.g., ref 65). In the case of a substratesupported lipid monolayer, however, a lateral compression, which would becaused by a dehydration of the monol ayer headgroups, would indeed be accompanied by a dewetting process. I t is likely that (duetothemuch higher spreading power compared to a bilayer on a bilayer) dewetting is energetically so unfavorablethat lateral-compression-and humidity-induced phase transitions in solid-supported monolayers are frustrated.

As already mentioned in the Introduction, the selfhealing properties of Langmuir-Blodgett fil ms might be of considerableimportancefor practical applications. The spreading power of bilayers on solid substrates is much lower compared to that of monolayers; therefore, the healing of monolayer defects before a second monolayer is deposited could be important for fabricating highly insulating bilayers.

(64) Parsegian, V. A.; Fuller, N.; Rand, R. P. Proc. Natl. Acad. Sci. U.S.A. 1979, 76, 2750-2754.

(65) Binder, H.; Gutberlet, T.; Anikin, A.; Klose, G. Biophys. J . 1998 74, 1908-1923. 


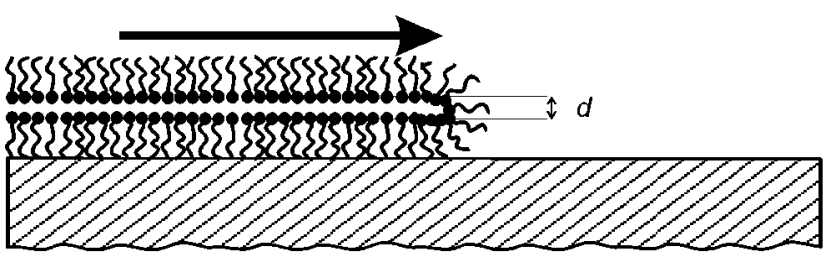

Figure 10. Possible experimental configuration for correlating monolayer spreading with the water content of the lipid/lipid interface, i.e., the thickness of the water layer d. Spreading is duetotheadvancement of theupper monolayer, whilethelower one is fixed on the substrate.

Many additionally important aspects of monolayer spreading could not be examined in the present work. Among these are a much more refined analysis of the quantitative influence of relative humi dity on spreading velocity, the use of solvent gases different from water, and a deeper analysis of roughness effects on spreading vel ocities.

Due to the asymmetric monolayer/support interface, the effect of monolayer hydration could not be distinguished from the swelling of the polymer support. Additionally, the monolayer/substrate separation in a hydrogel-supported membrane is difficult to determine, due to the swelling of the polymer cushion over length scales much larger than themonolayer/substrateinterface. These disadvantages could possibly be circumvented by spreading of a lipid monolayer on top of another monolayer, as depicted in Figure 10 for example. A hydrophobized substratewoul d be partially coated by Langmuir-Blodgett transfer. Subsequent withdrawal of the substrate would then result in an inverse bilayer (a solid-supported "foam fil $\mathrm{m}^{\prime \prime}$ ) with a defined bilayer edge. Substantially increasing $\mathrm{RH}$ should lead to a precisely measurable (e.g., by ellipsometry or surface plasmon resonance spectroscopy) change in the water layer thickness d (amounting to up to $\left.3 \mathrm{~nm}^{66}\right)$. Provided that thespreading front displacement is small compared to monolayer expansion, the spreading velocity should be determined by friction in thelipid/lipid interface lowered by repulsive hydration forces, and the spreading power. The water layer thickness in the case of monolayers with low lateral pressure, however, is determined not only by repul sivehydration forces but also by attractive hydrophobic forces as has been shown in the case of partially depleted bilayers by $\mathrm{Helm}$ et al., ${ }^{67}$ so additional phenomena can be studied.

Acknowledgment. Wethank Wolfgang Knoll for his generous support within the project and Diethelm J ohannsmann for the donation of the apparatus for controlling relativehumidities. Andreas Scheller is acknowledged for his hel $p$ with the computer program, which controlled the humidity apparatus.

\section{LA0255673}

(66) Rand, R. P.; Parsegian, V. A. Biochim. Biophys. Acta 1989, 988 $351-376$.

(67) Helm, C. A.; I sraelachvili, J . N.; McGuiggan, P. M. Science1989, 246, 919-922. 